\title{
Construction of Substituted Benzenes via Pd-Catalyzed Cross-Coupling/ Cyclization Reaction of Vinyl halides and Terminal Alkynes
}

\author{
Meihua Xie*, Shengke Wang, Jun Wang, Kuang Fang, Changqing Liu, \\ Chao Zha, Jing Jia \\ Key Laboratory of Functional Molecular Solids (Ministry of Education), Anhui Key Laboratory of \\ Molecule-Based Materials, College of Chemistry and Materials Science, Anhui Normal University, Wuhu, 241000, \\ China
}

\section{Supporting Information}

1. X-ray crystallographic data for 2-benzyl-6-( $p$-tolylsulfonyl)biphenyl (3a)......S2-3

2. ${ }^{1} \mathrm{H}$ NMR and ${ }^{13} \mathrm{C}$ NMR spectra of the products...........................S4-27 


\section{X-ray crystallographic data for 2-benzyl-6-(p-tolylsulfonyl)biphenyl (3a)}

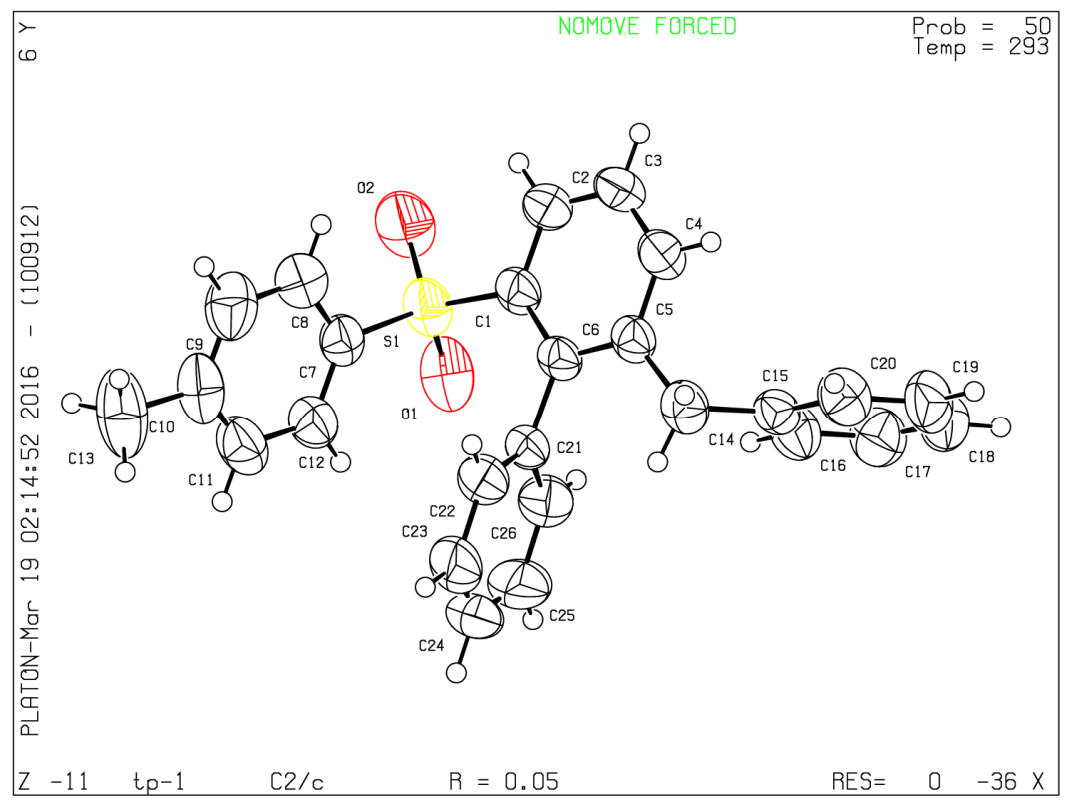

ORTEP representation of 3a showing 30\% probability ellipsoids

Crystals of compound 3a suitable for x-ray analysis were obtained by recrystallization from ethyl acetate/hexane. The crystal structure has been deposited at the Cambridge Crystallographic Data Centre (CCDC 1436225). The data can be obtained free of charge via the Internet at www.ccdc.cam.ac.uk/data request/cif.

Table 1. Crystal data and structure refinement for $\mathbf{3 a}$.

Identification code

Empirical formula

Formula weight

Temperature

Wavelength

Crystal system, space group

Unit cell dimensions 3a

$\mathrm{C}_{26} \mathrm{H}_{22} \mathrm{O}_{2} \mathrm{~S}$

398.50

293(2) K

$0.71073 \AA$

Monoclinic, $\mathrm{C} 2 / \mathrm{c}$

$$
\begin{array}{ll}
\mathrm{a}=34.459(3) \AA & \alpha=90^{\circ} . \\
\mathrm{b}=6.7887(7) \AA \quad \alpha=93.152(2)^{\circ} . & \\
\mathrm{c}=18.2939(18) \AA \quad & \gamma=90^{\circ} .
\end{array}
$$


Volume

Z, Calculated density

Absorption coefficient

$\mathrm{F}(000)$

Crystal size

Theta range for data collection

Limiting indices

Reflections collected / unique

Completeness to theta $=27.68$

Absorption correction

Max. and min. transmission

Refinement method

Data / restraints / parameters

Goodness-of-fit on $\mathrm{F}^{\wedge} 2$

Final $\mathrm{R}$ indices [I $>2$ sigma(I)]

$\mathrm{R}$ indices (all data)

Extinction coefficient

Largest diff. peak and hole
4273.1(7) $\AA^{3}$

8, $1.239 \mathrm{Mg} / \mathrm{m}^{3}$

$0.170 \mathrm{~mm}^{-1}$

1680

$0.18 \times 0.16 \times 0.15 \mathrm{~mm}$

2.23 to $27.68^{\circ}$

$-44<=\mathrm{h}<=44,-8<=\mathrm{k}<=8,-23<=1<=22$

$17852 / 4951[\mathrm{R}(\mathrm{int})=0.0282]$

$99.2 \%$

None

0.9749 and 0.9700

Full-matrix least-squares on $\mathrm{F}^{2}$

$4951 / 0$ / 264

1.023

$\mathrm{R} 1=0.0464, \mathrm{wR} 2=0.1356$

$\mathrm{R} 1=0.0630, \mathrm{wR} 2=0.1510$

$0.0068(5)$

0.212 and -0.310 e. $\mathrm{A}^{-3}$ 


\section{2. ${ }^{1} \mathrm{H}$ NMR and ${ }^{13} \mathrm{C}$ NMR spectra of the products}

2-benzyl-6-(4-tolylsulfonyl)biphenyl (3a)
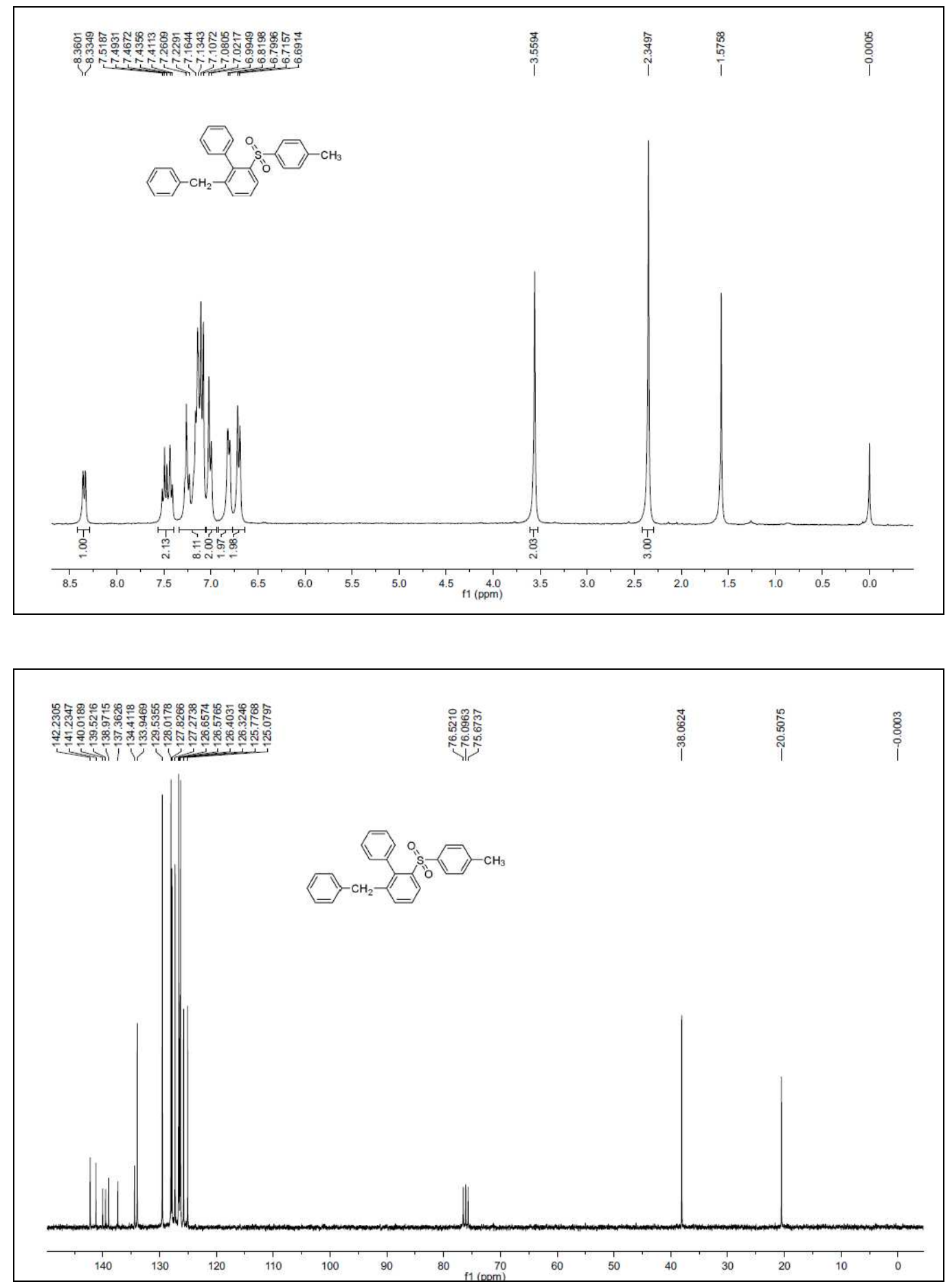
2-(4-methylbenzyl)-6-(4-tolylsulfonyl)biphenyl (3b)
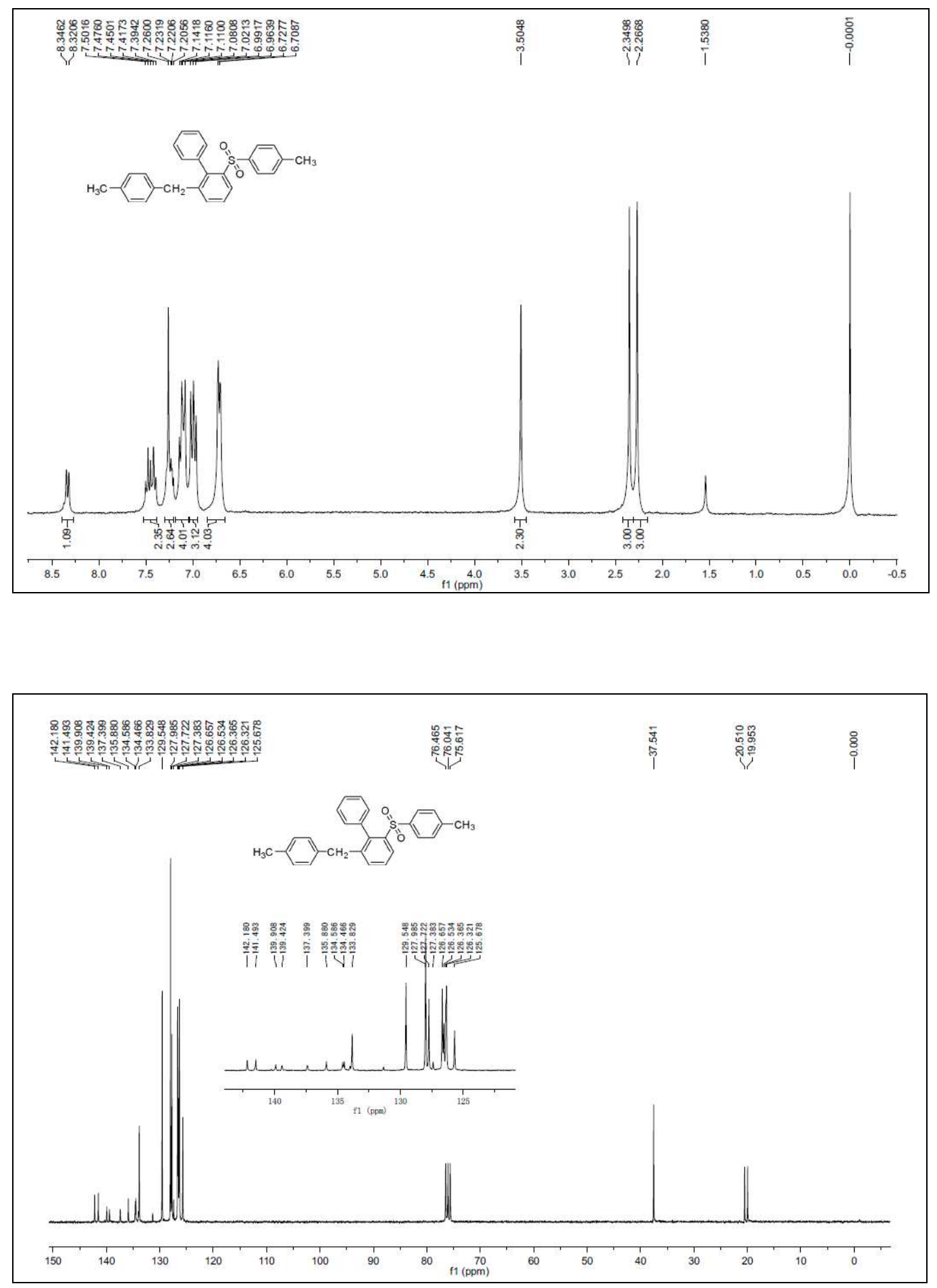
2-(4-fluorobenzyl)-6-(4-tolylsulfonyl)biphenyl (3c)
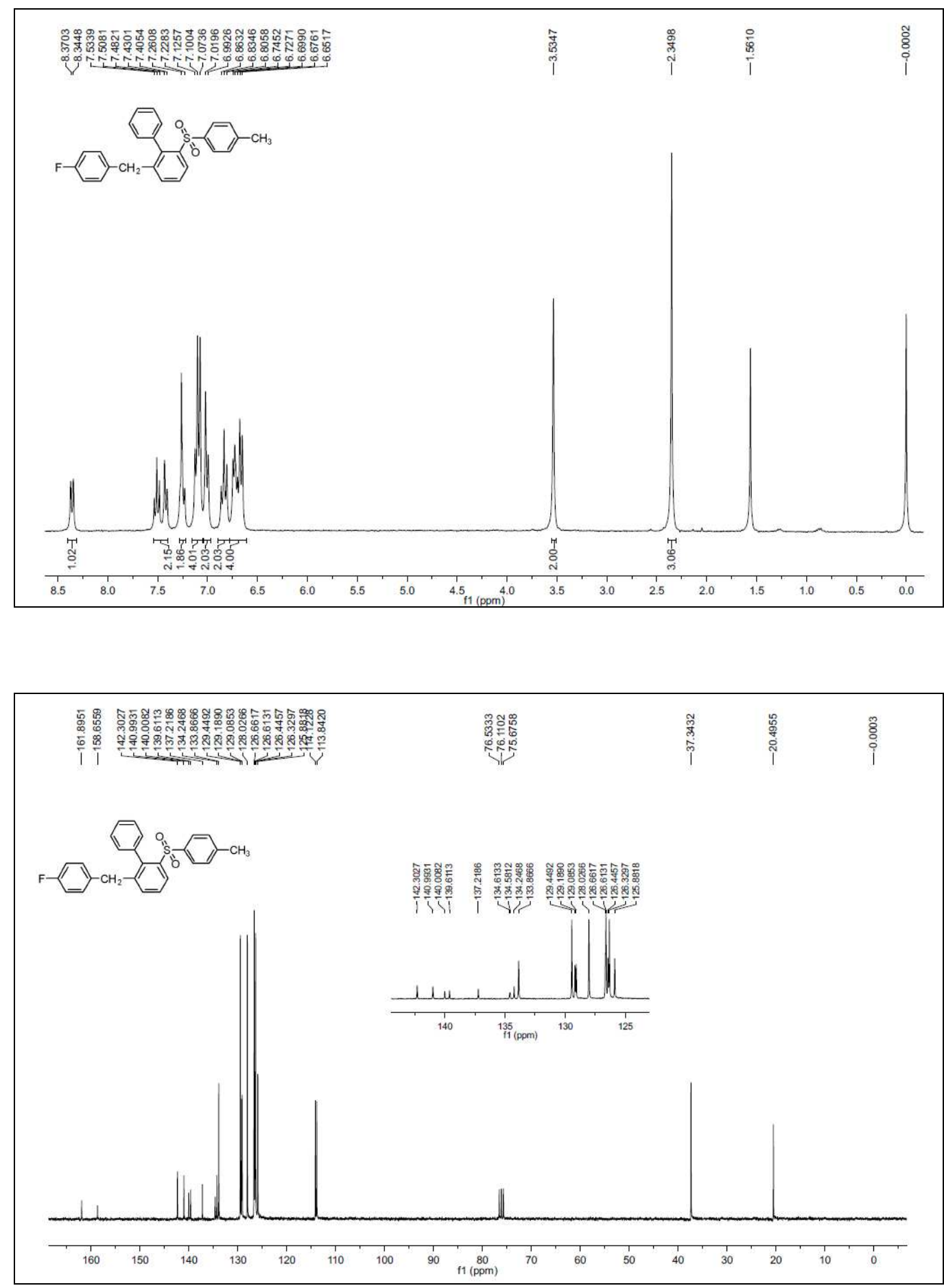
2-hexyl-6-tosylbiphenyl (3d)
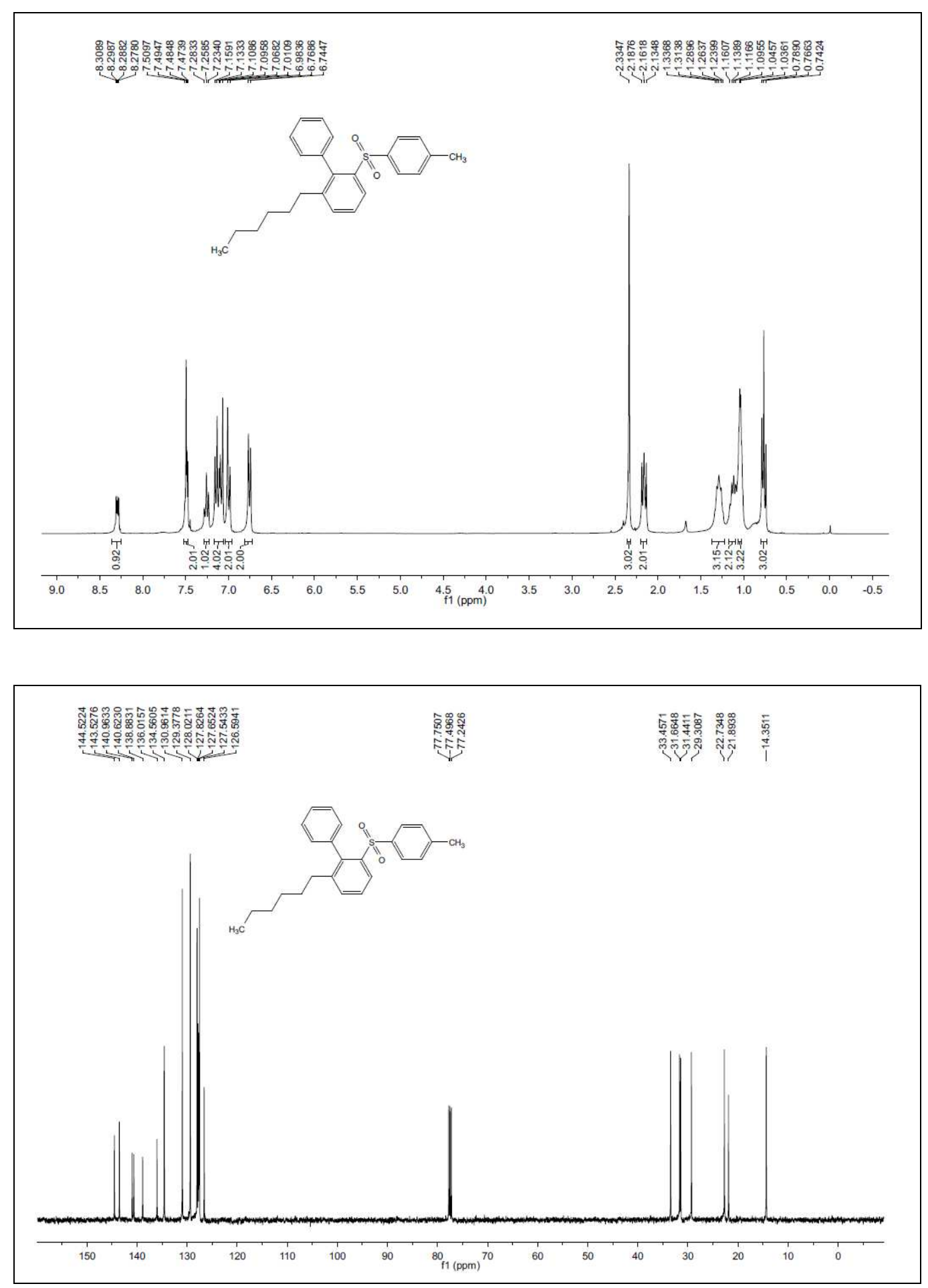
2-(cyclopropylmethyl)-6-(4-tolylsulfonyl)biphenyl (3e)
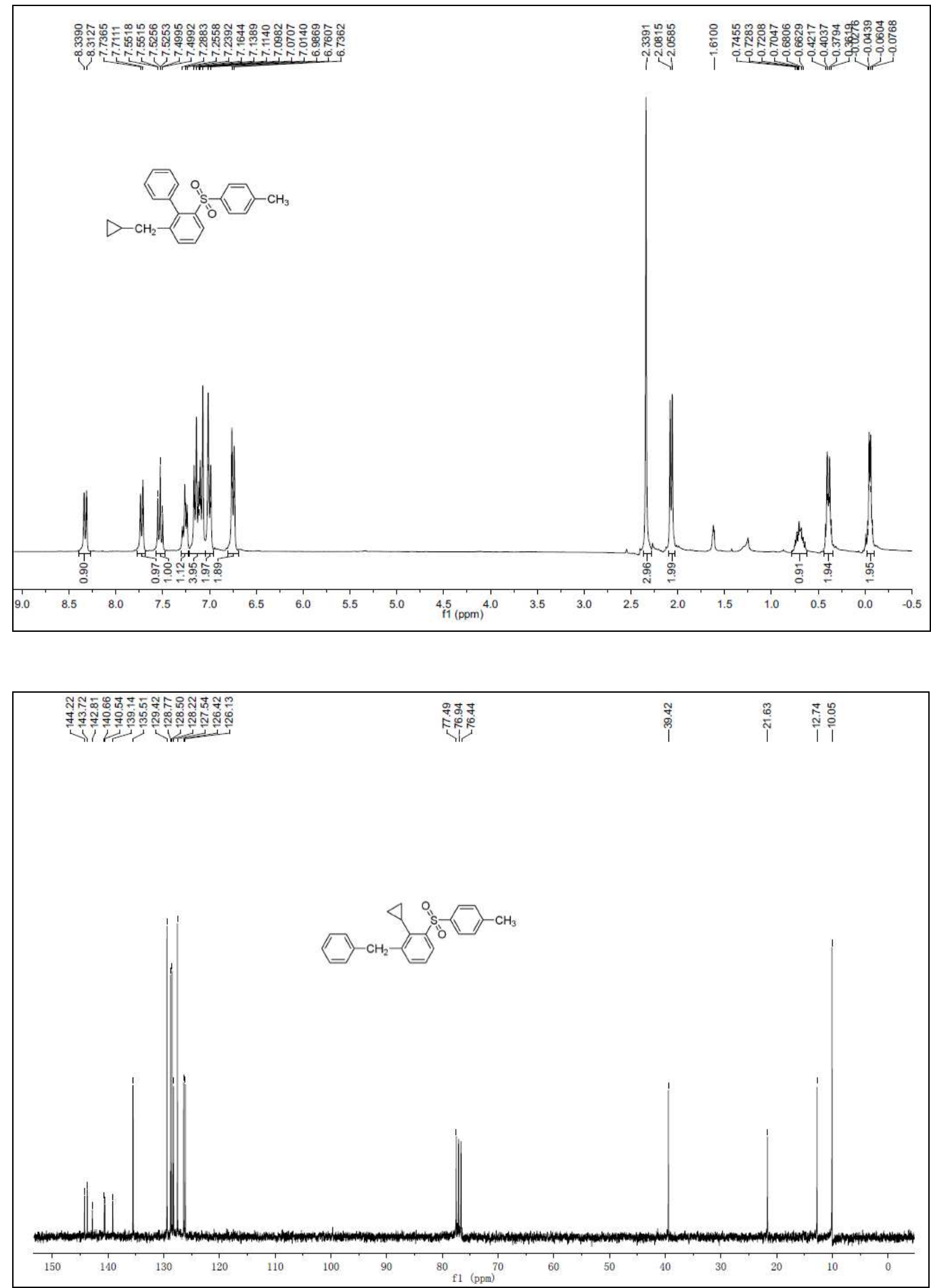
2-neopentyl-6-(4-tolylsulfonyl)biphenyl (3f)
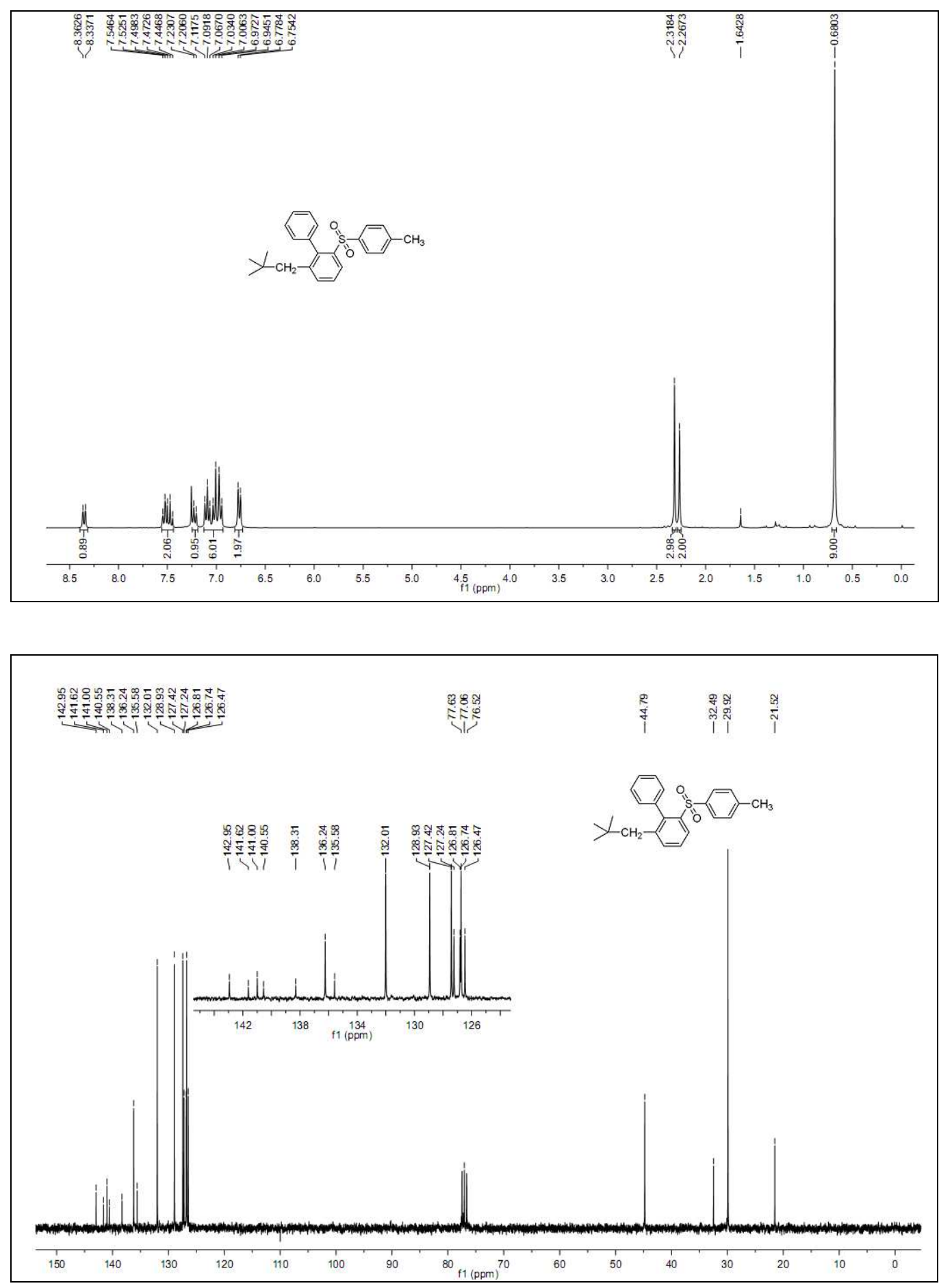
2-benzyl-4-methyl-6-(4-tolylsulfonyl)biphenyl (3g)
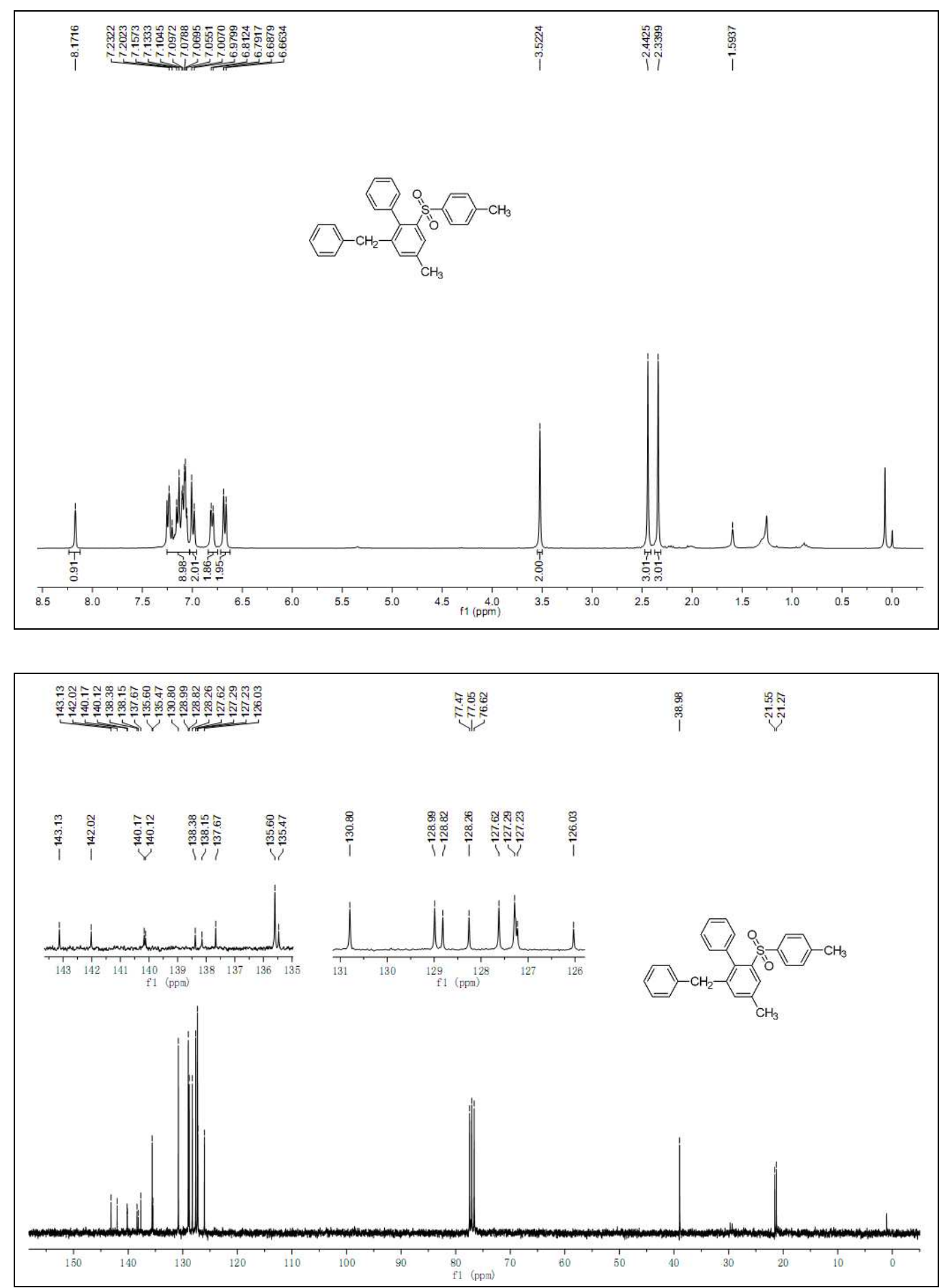
2-benzyl-4'-methyl-6-(4-tolylsulfonyl)biphenyl (3h)
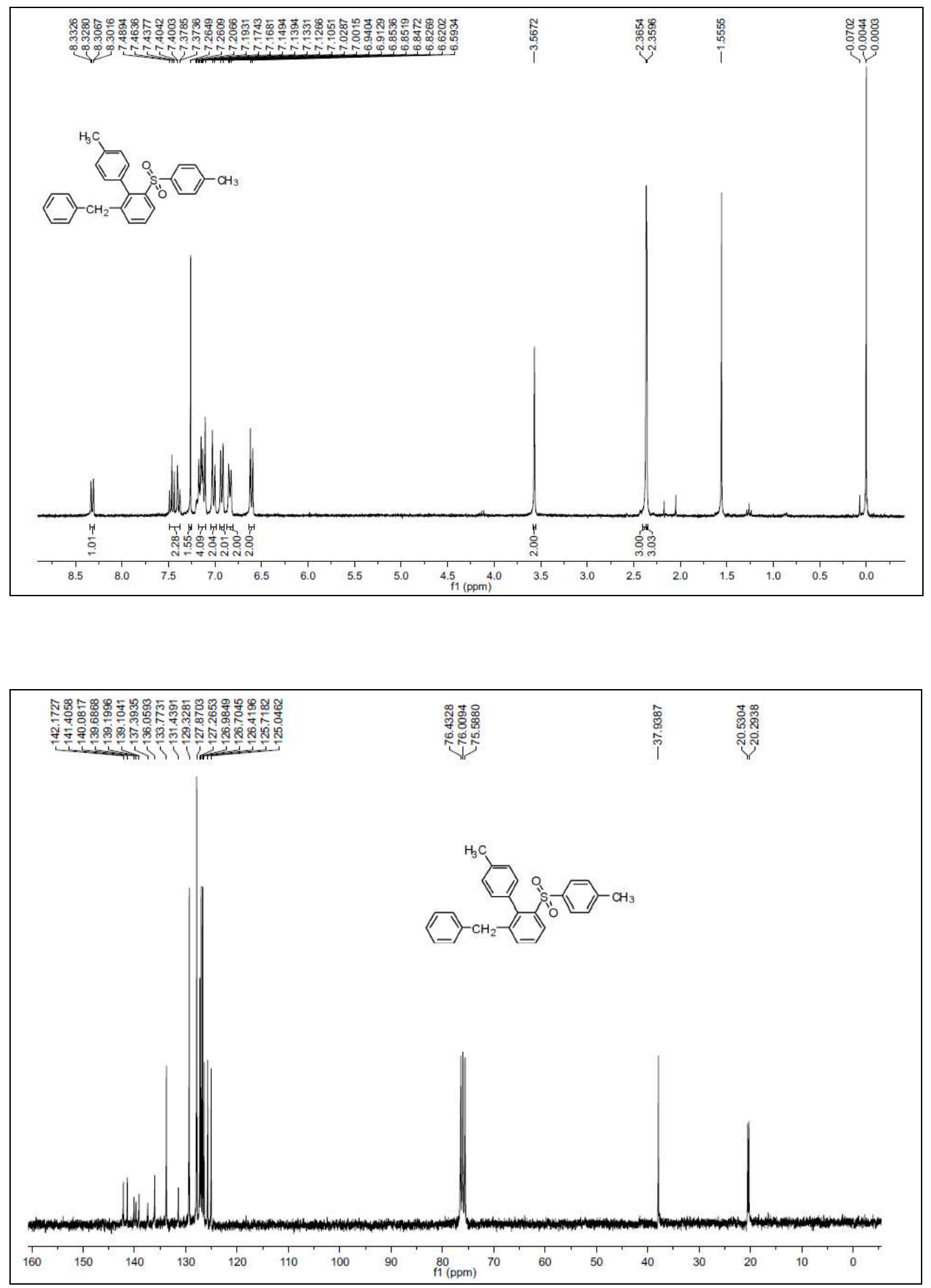
4'-methyl-2-(4-methylbenzyl)-6-(4-tolylsulfonyl)biphenyl (3i)
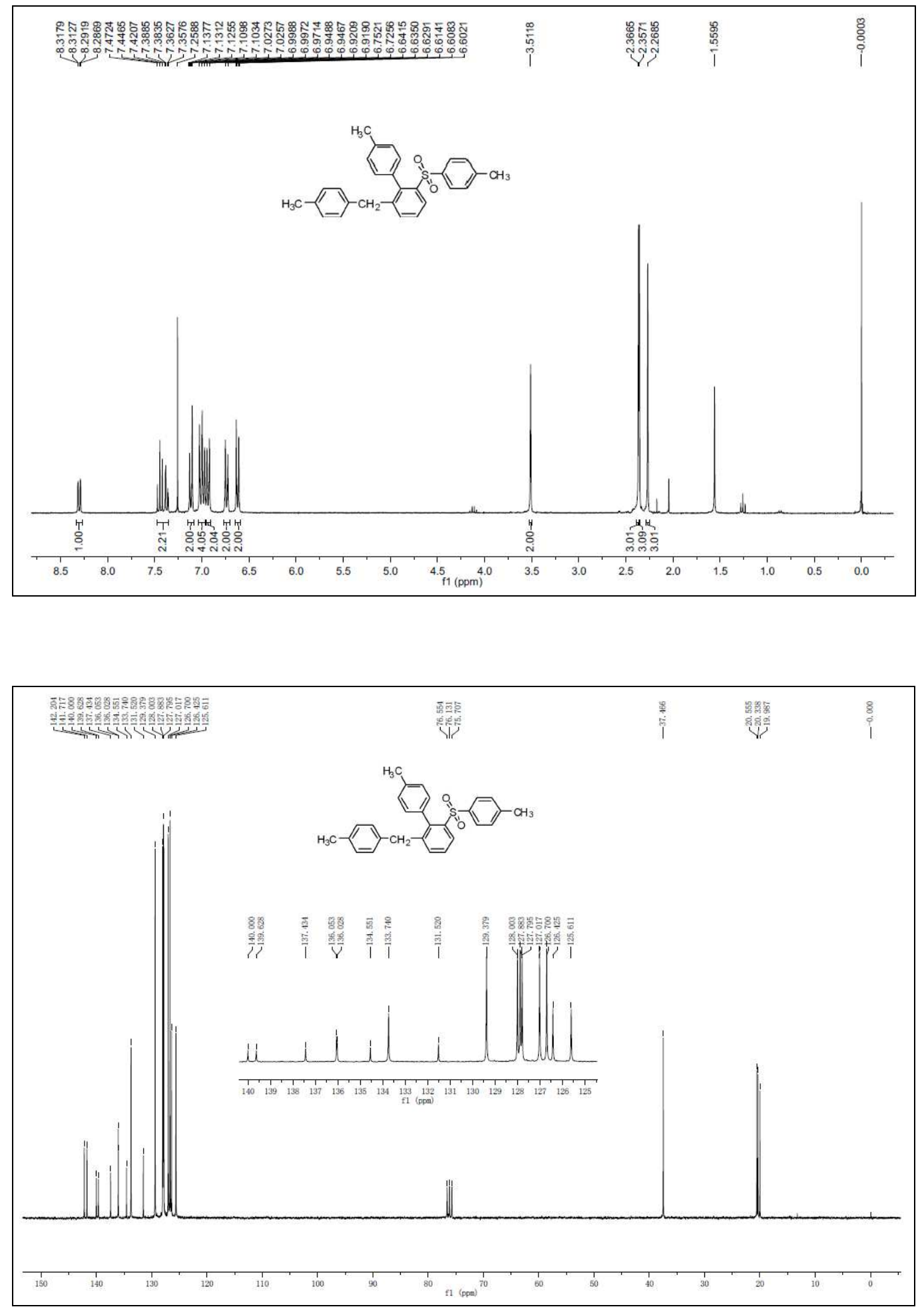
2-(4-fluorobenzyl)-4'-methyl-6-(4-tolylsulfonyl)biphenyl (3j)
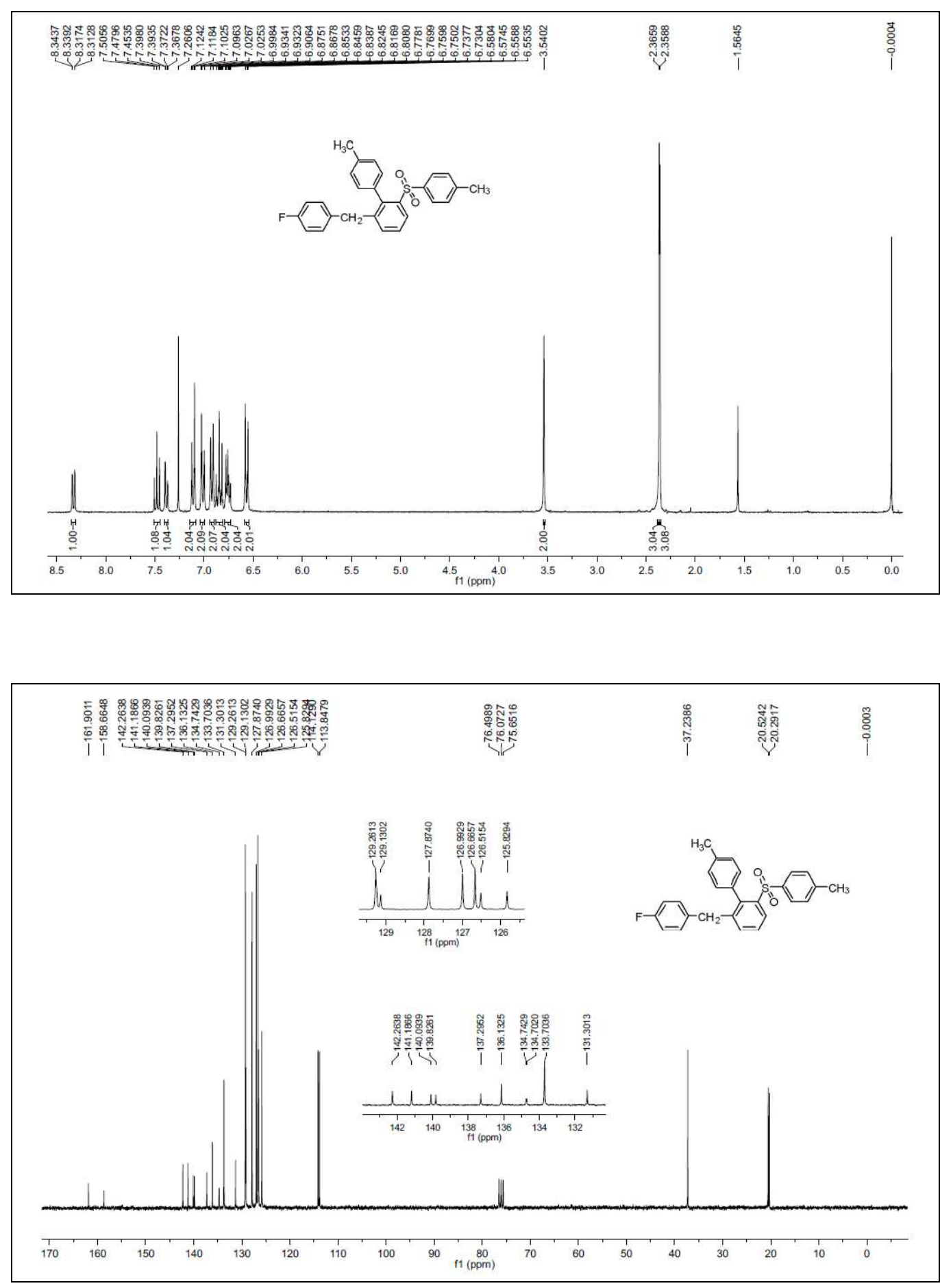
2-benzyl-4'-fluoro-6-(4-tolylsulfonyl)biphenyl (3k)
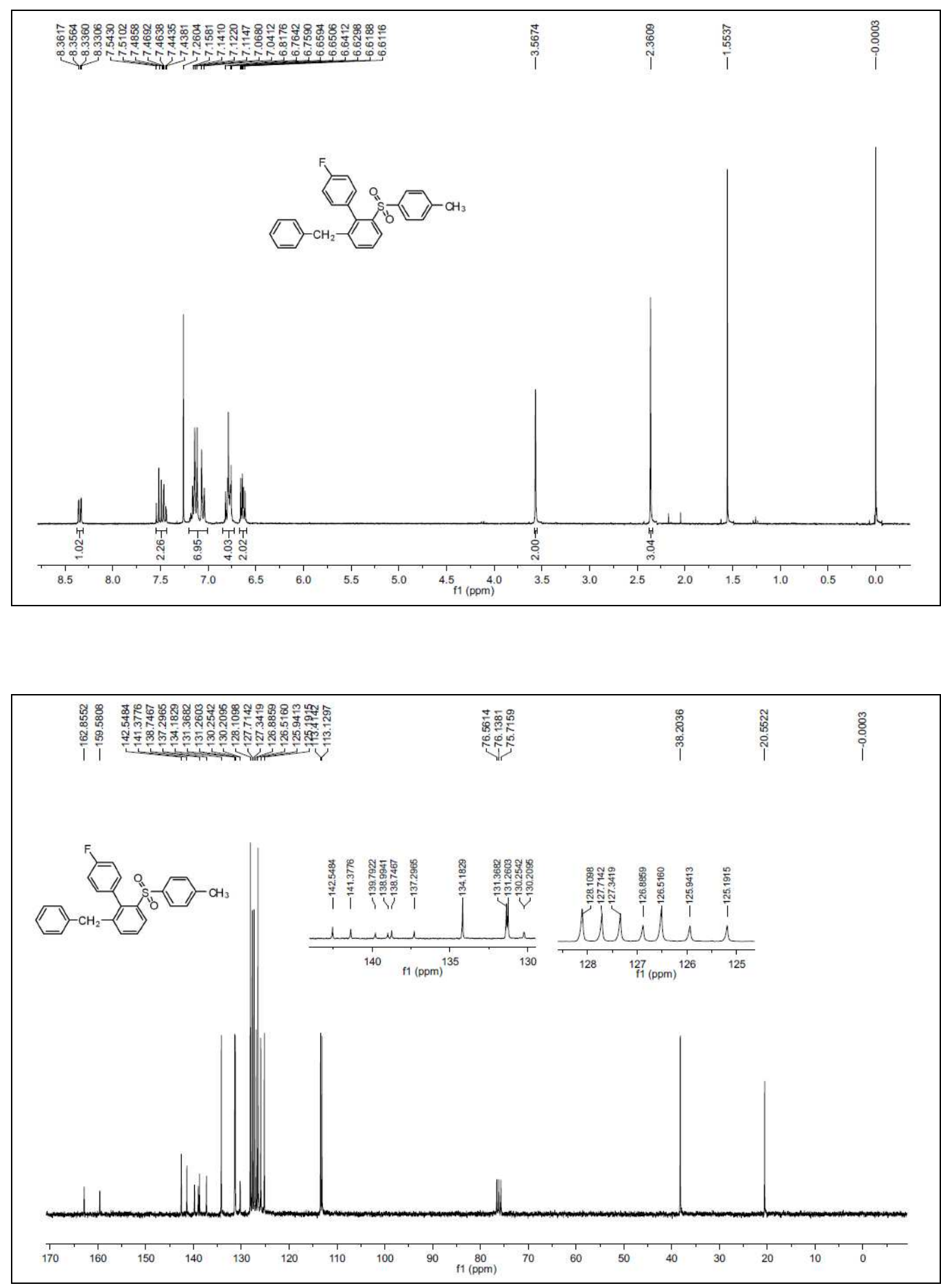
4'-fluoro-2-(4-methylbenzyl)-6-(4-tolylsulfonyl)biphenyl (31)
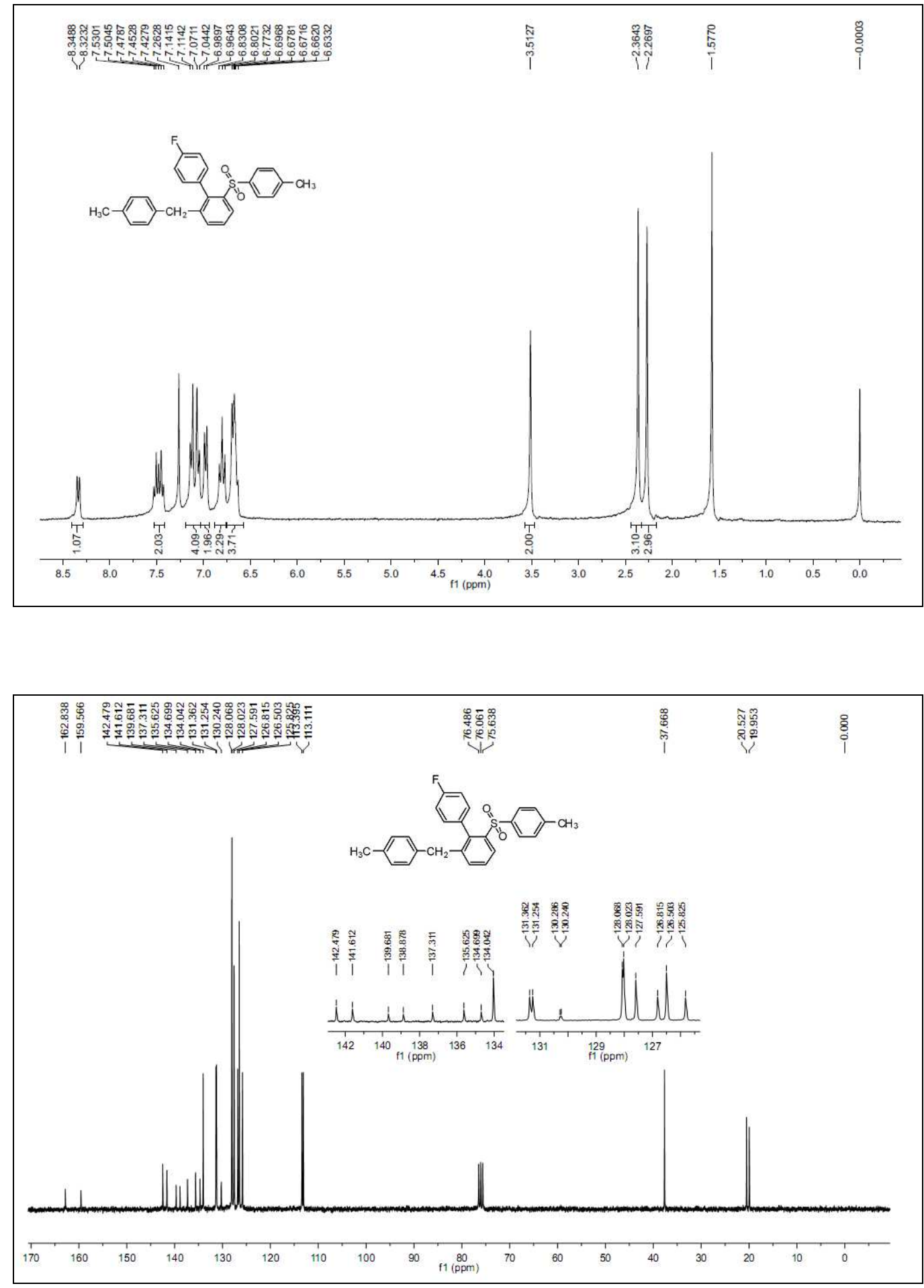
4'-fluoro-2-(4-fluorobenzyl)-6-(4-tolylsulfonyl)biphenyl (3m)
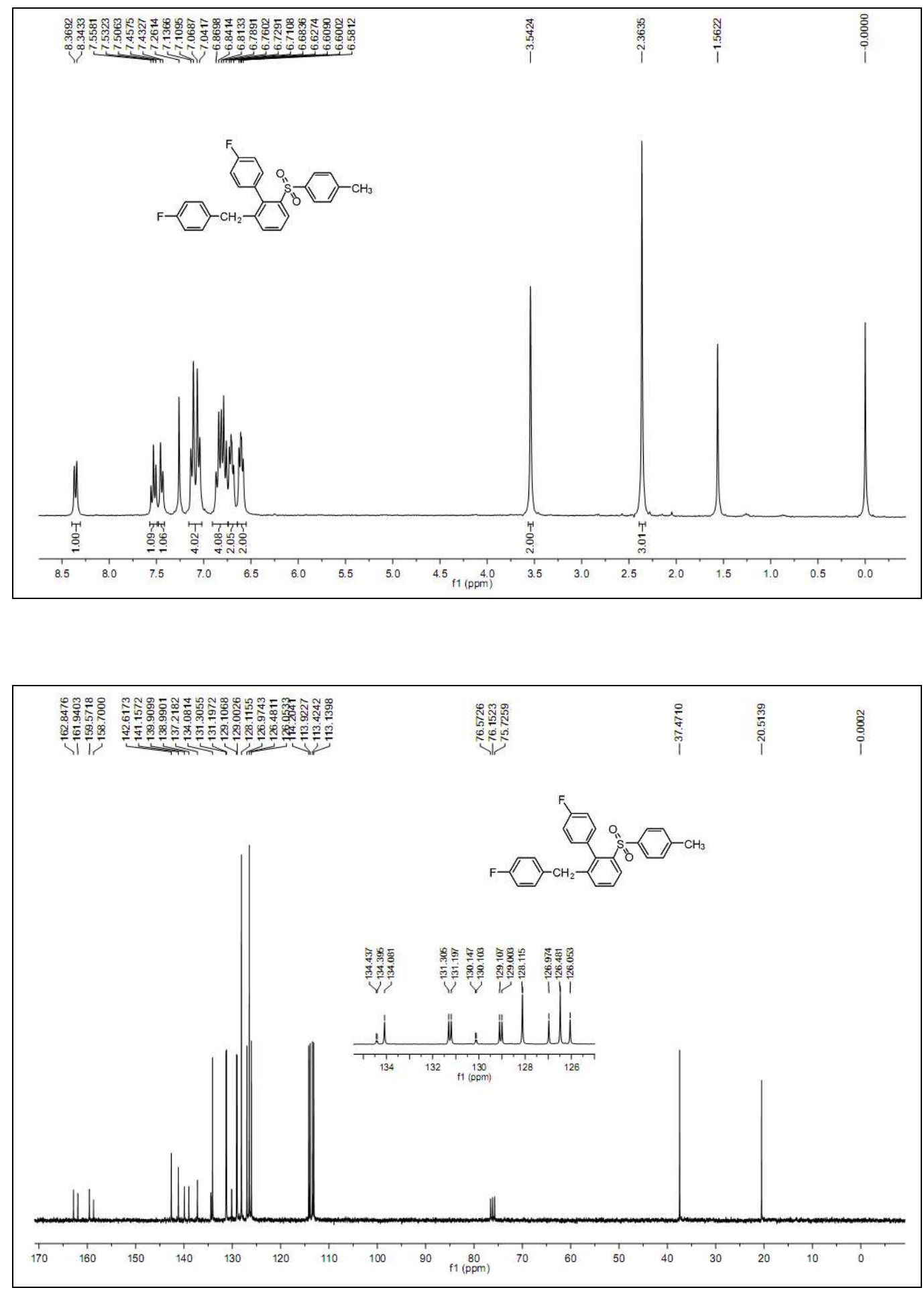
1-benzyl-2-pentyl-3-(4-tolylsulfonyl)benzene (3n)
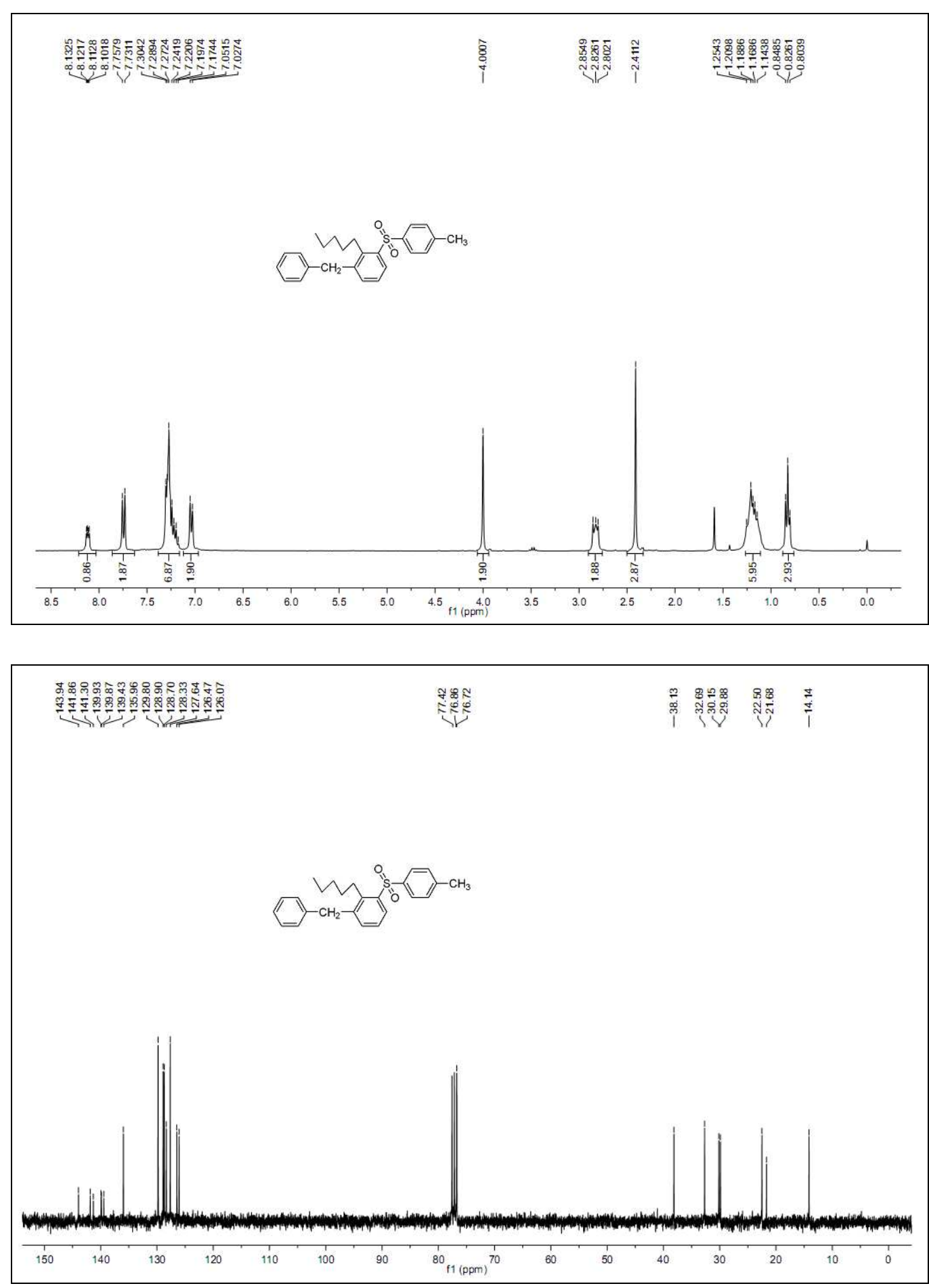
1-benzyl-2-cyclopropyl-3-(4-tolylsulfonyl)benzene (3o)
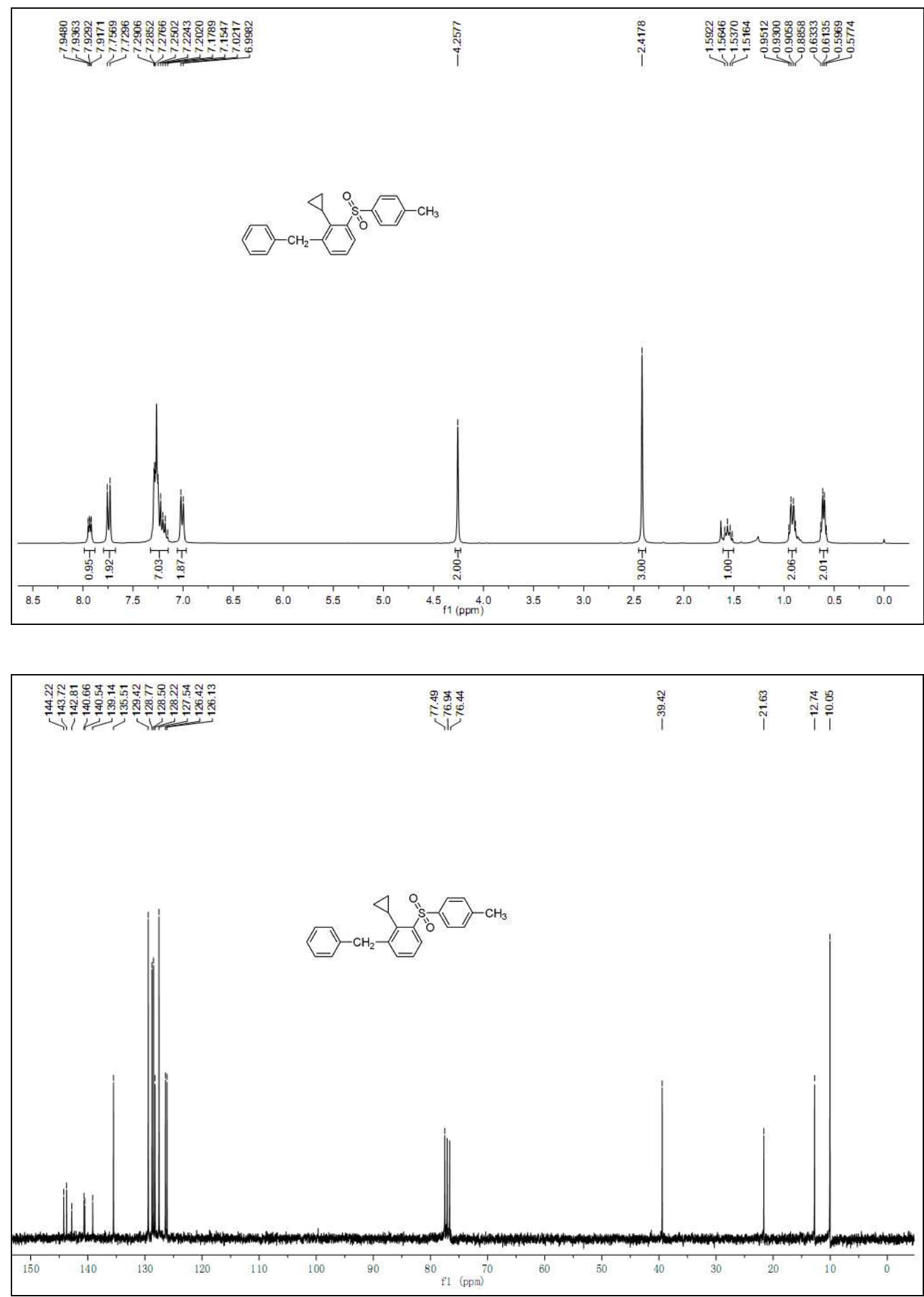
2-benzyl-6-(4-methylbenzoyl)biphenyl (5a)
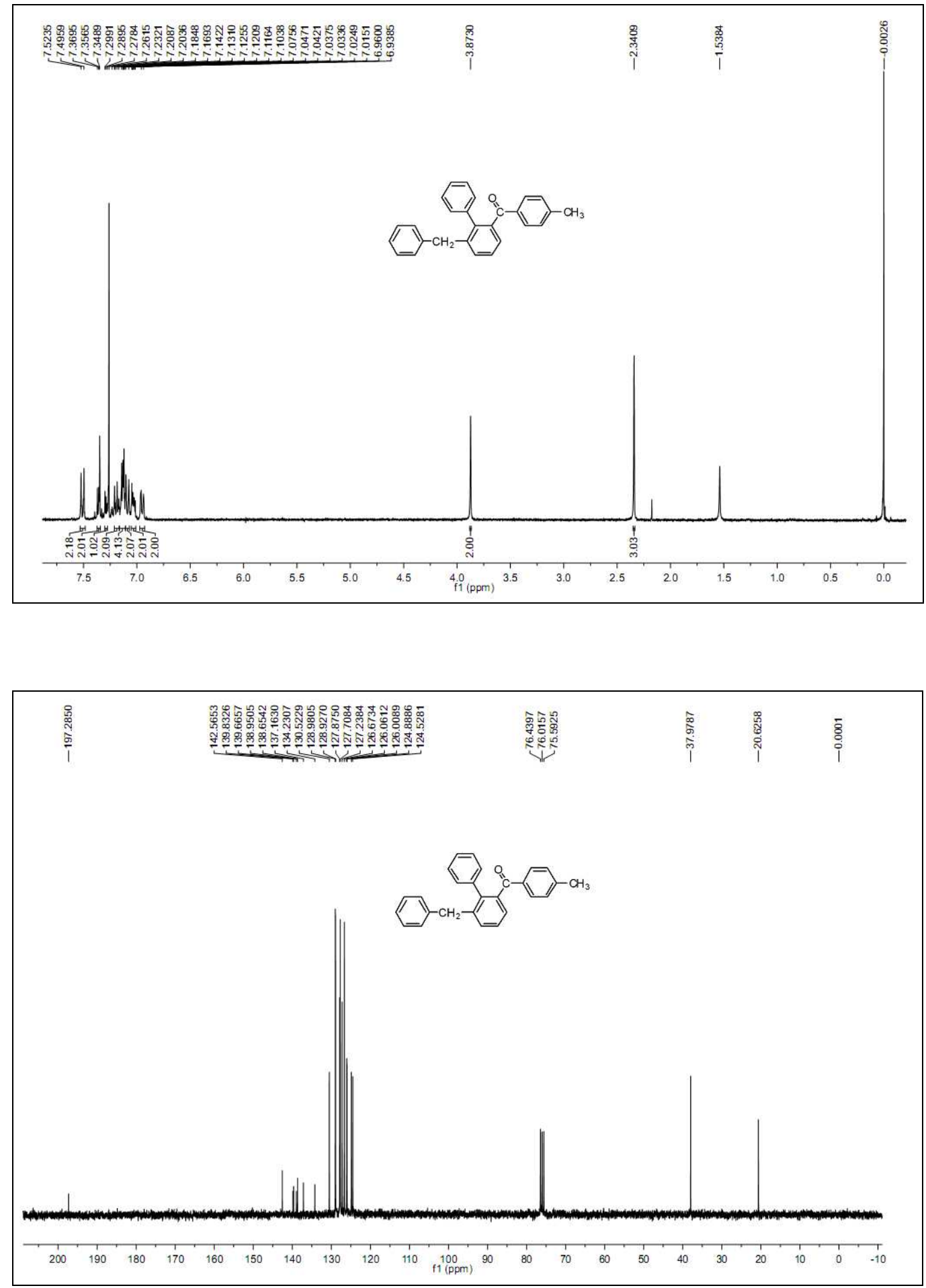
2-(4-methylbenzyl)-6-(4-methylbenzoyl)biphenyl (5b)
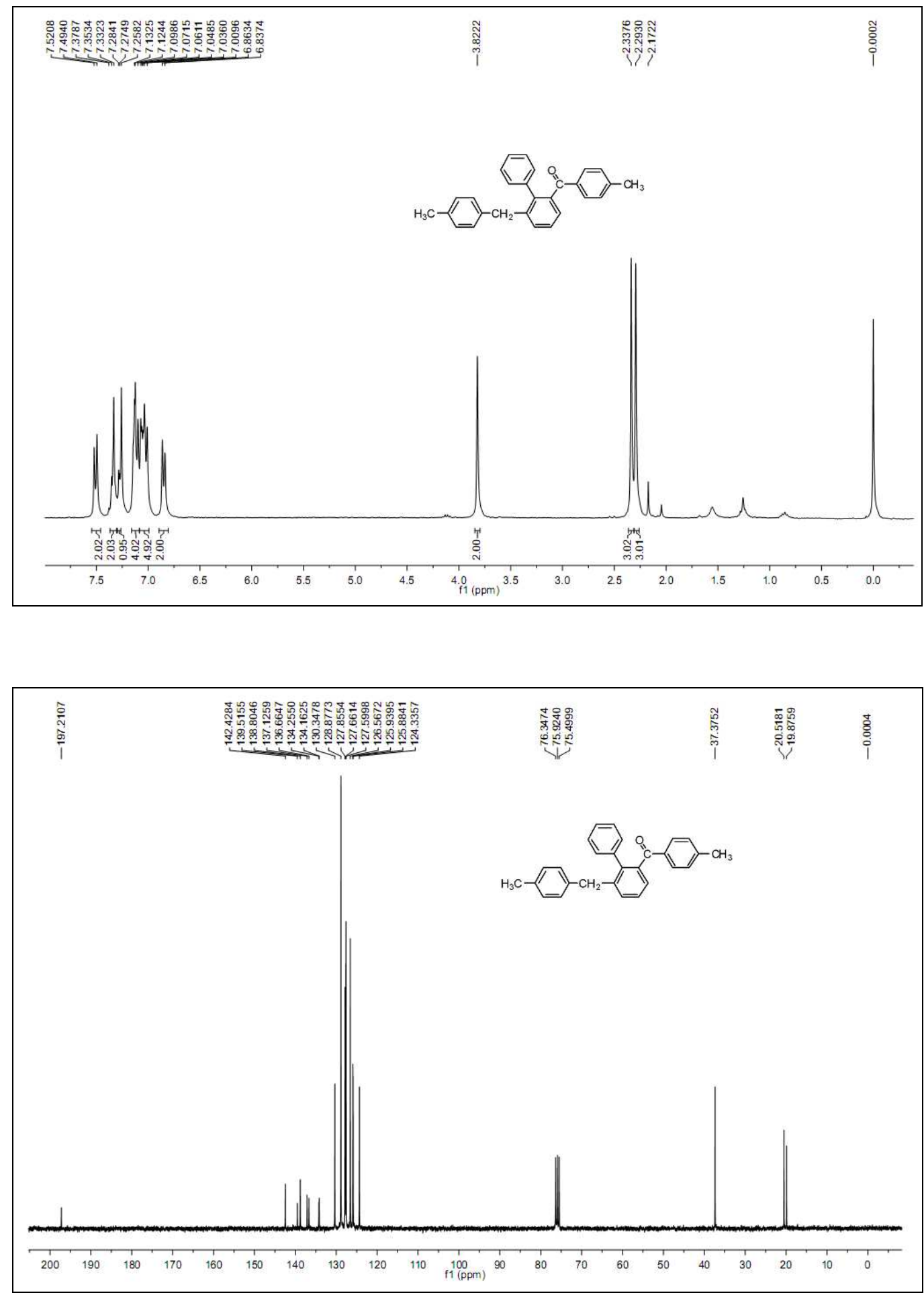
2-(4-fluorobenzyl)-6-(4-methylbenzoyl)biphenyl (5c)
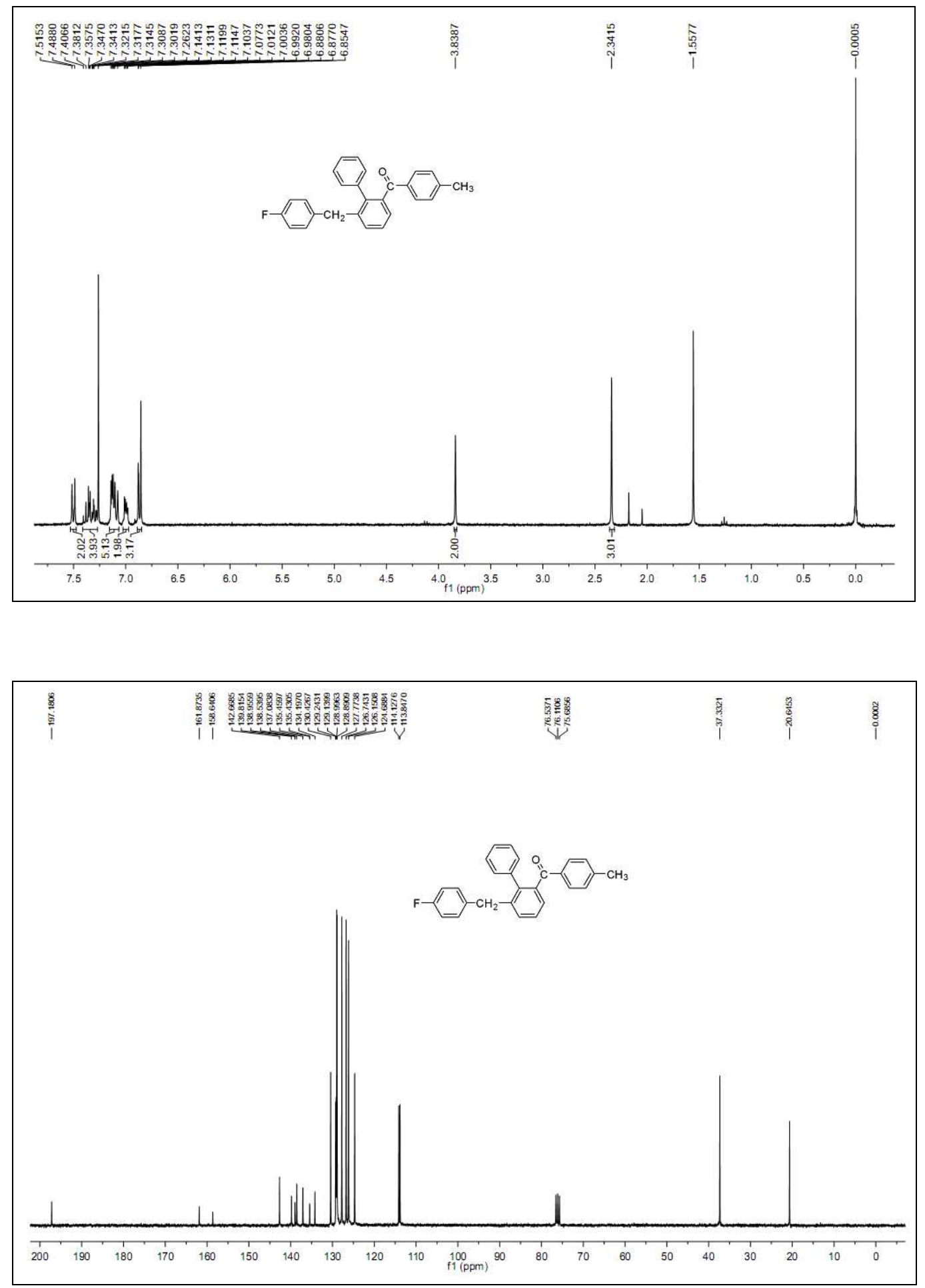
2-(cyclopropylmethyl)-6-(4-methylbenzoyl)biphenyl (5d)
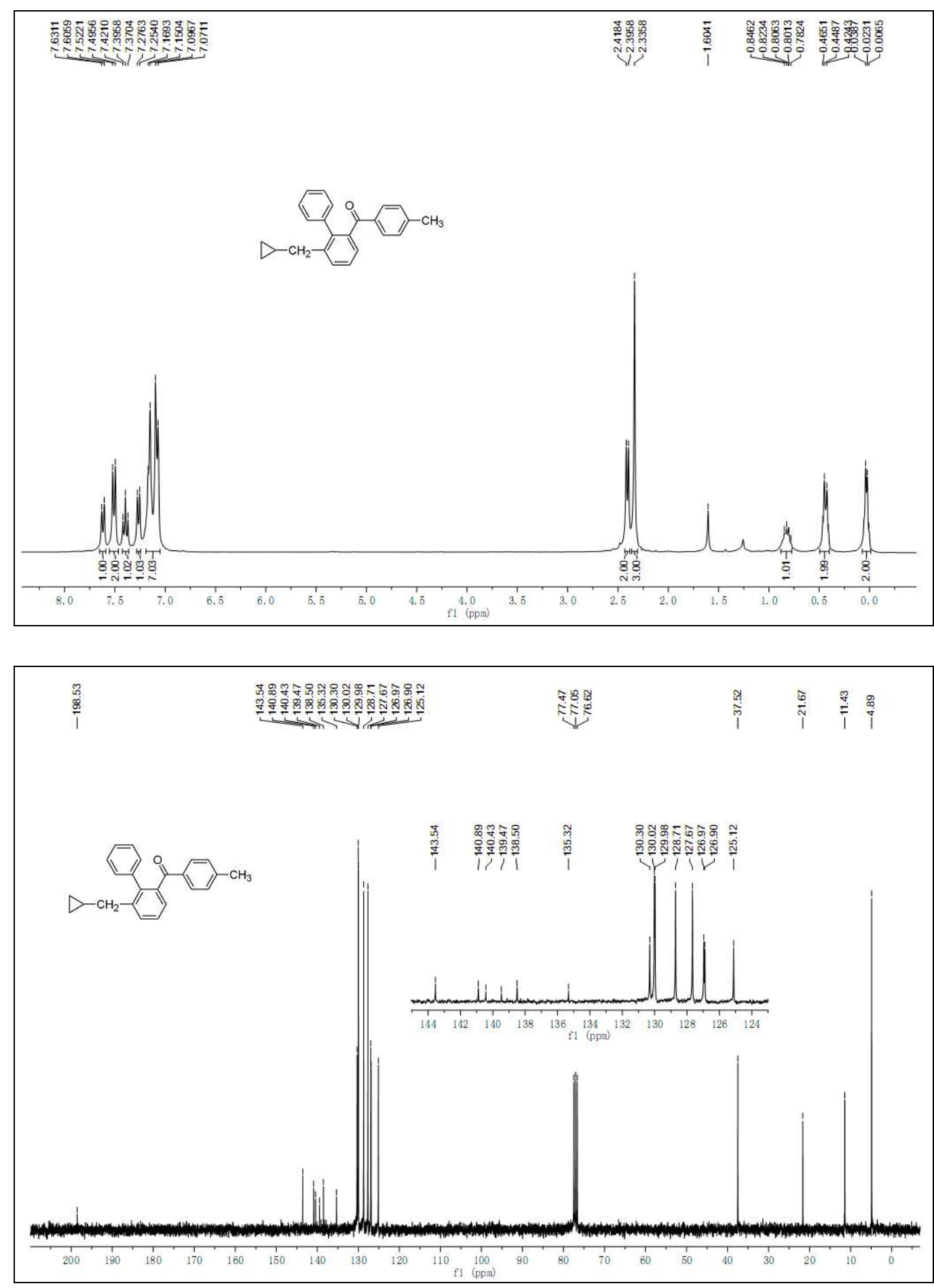
6-butyryl-2-benzylbiphenyl (5e)
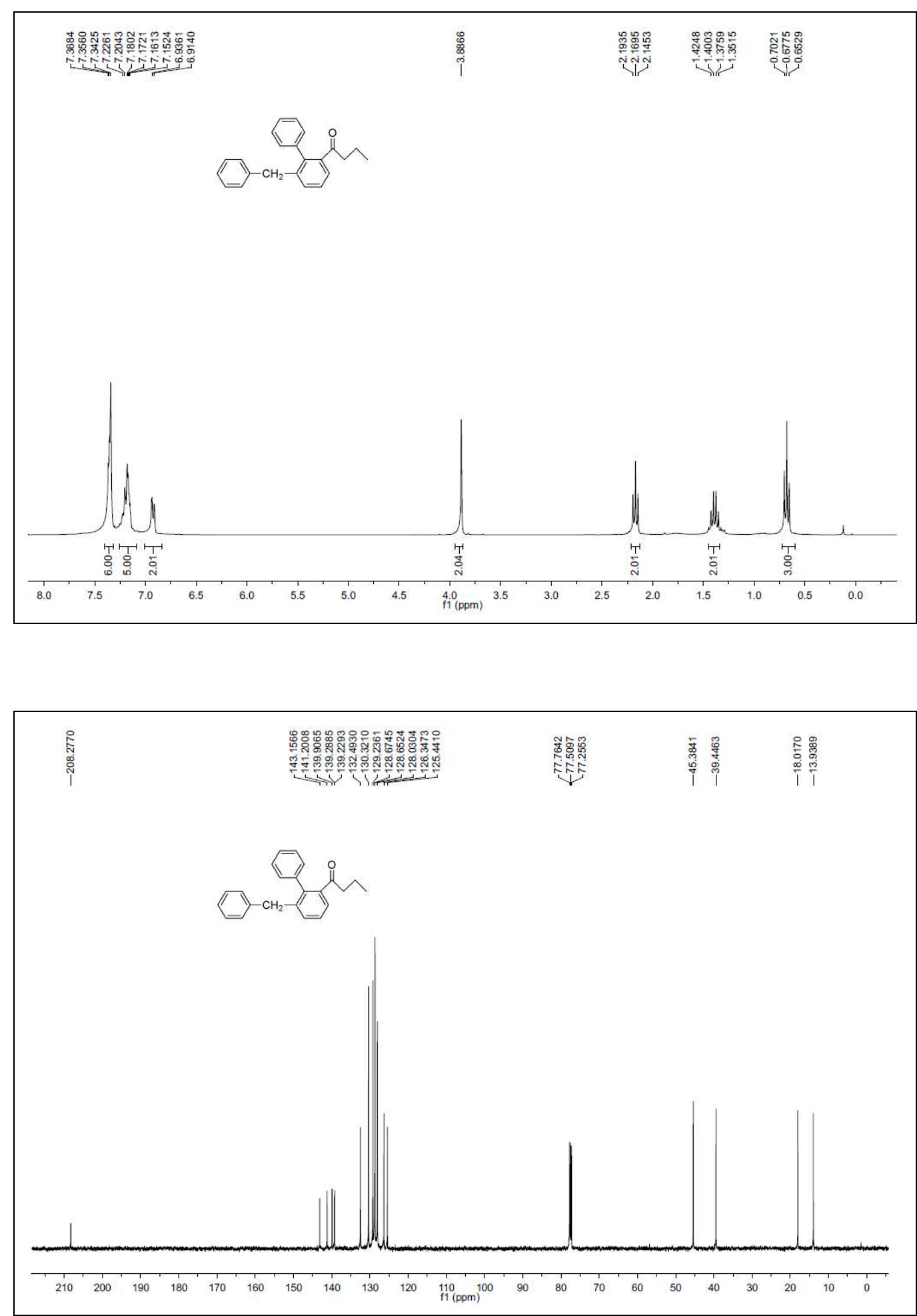
2-benzyl-4'-methyl-6-(4-chlorobenzoyl)biphenyl (5f)
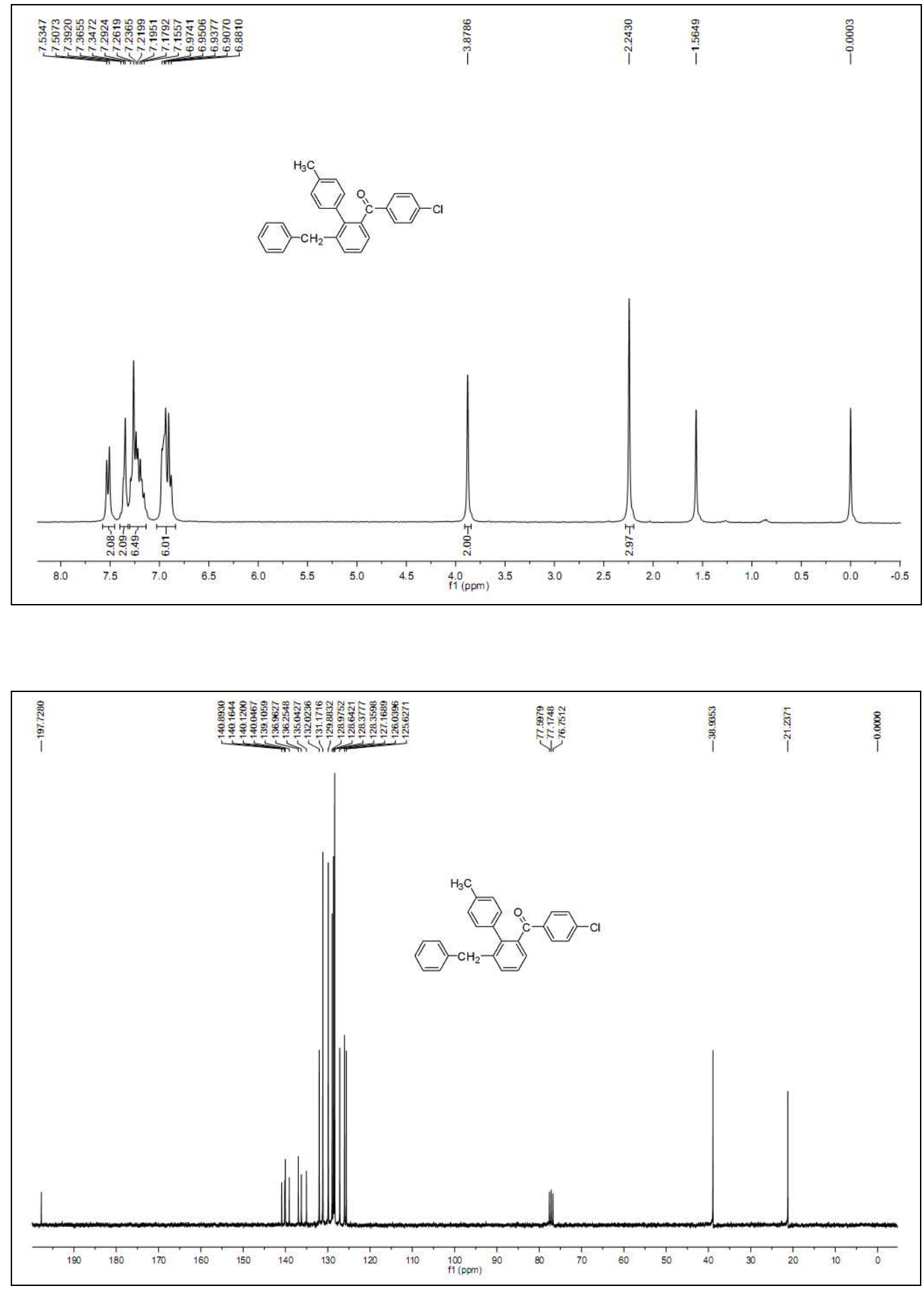
2-benzyl-4'-methyl-6-(4-nitrobenzoyl)biphenyl (5g)
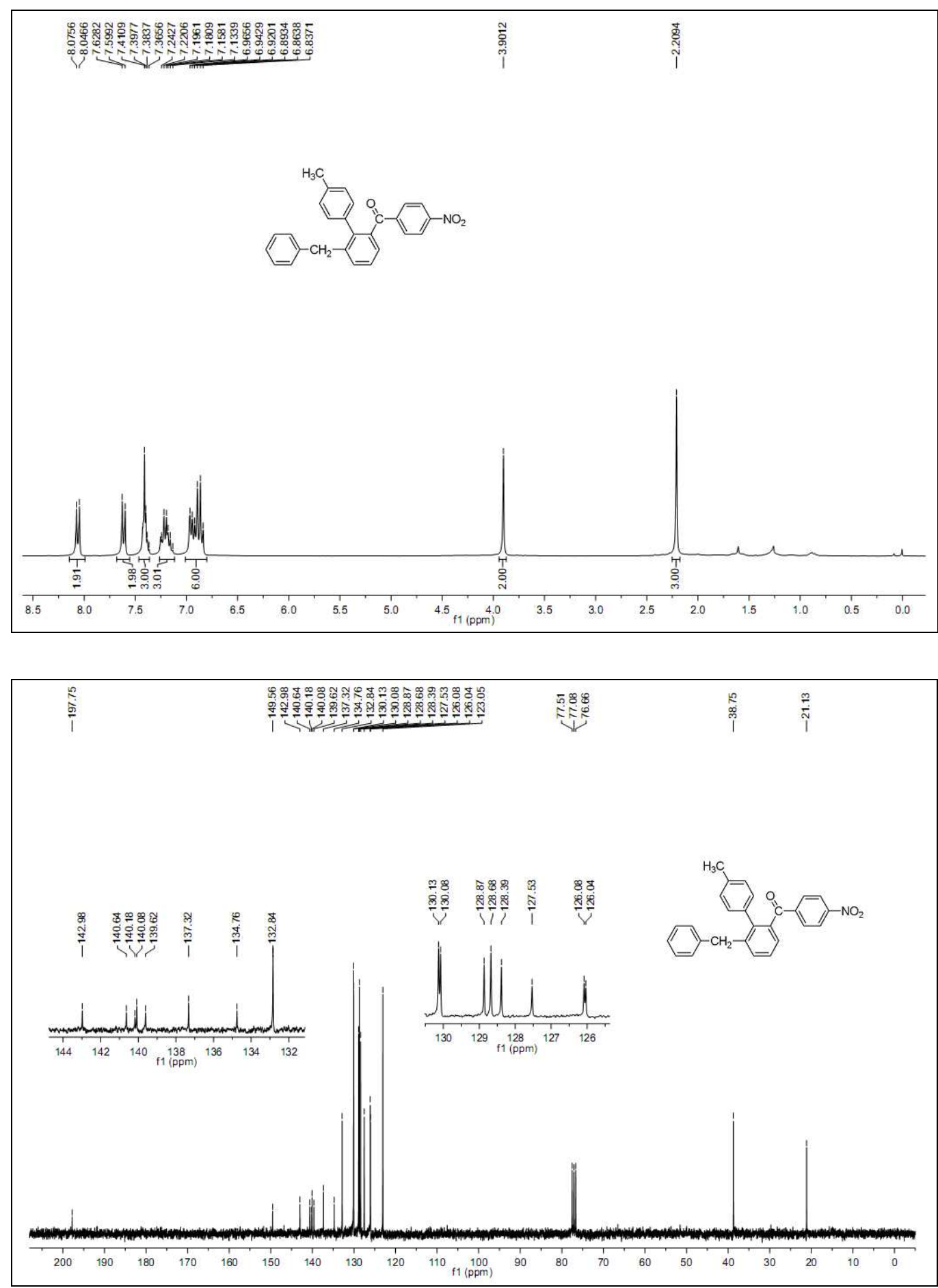
2-(4-methylbenzyl)-4'-methyl-6-(4-chlorobenzoyl)biphenyl (5h)
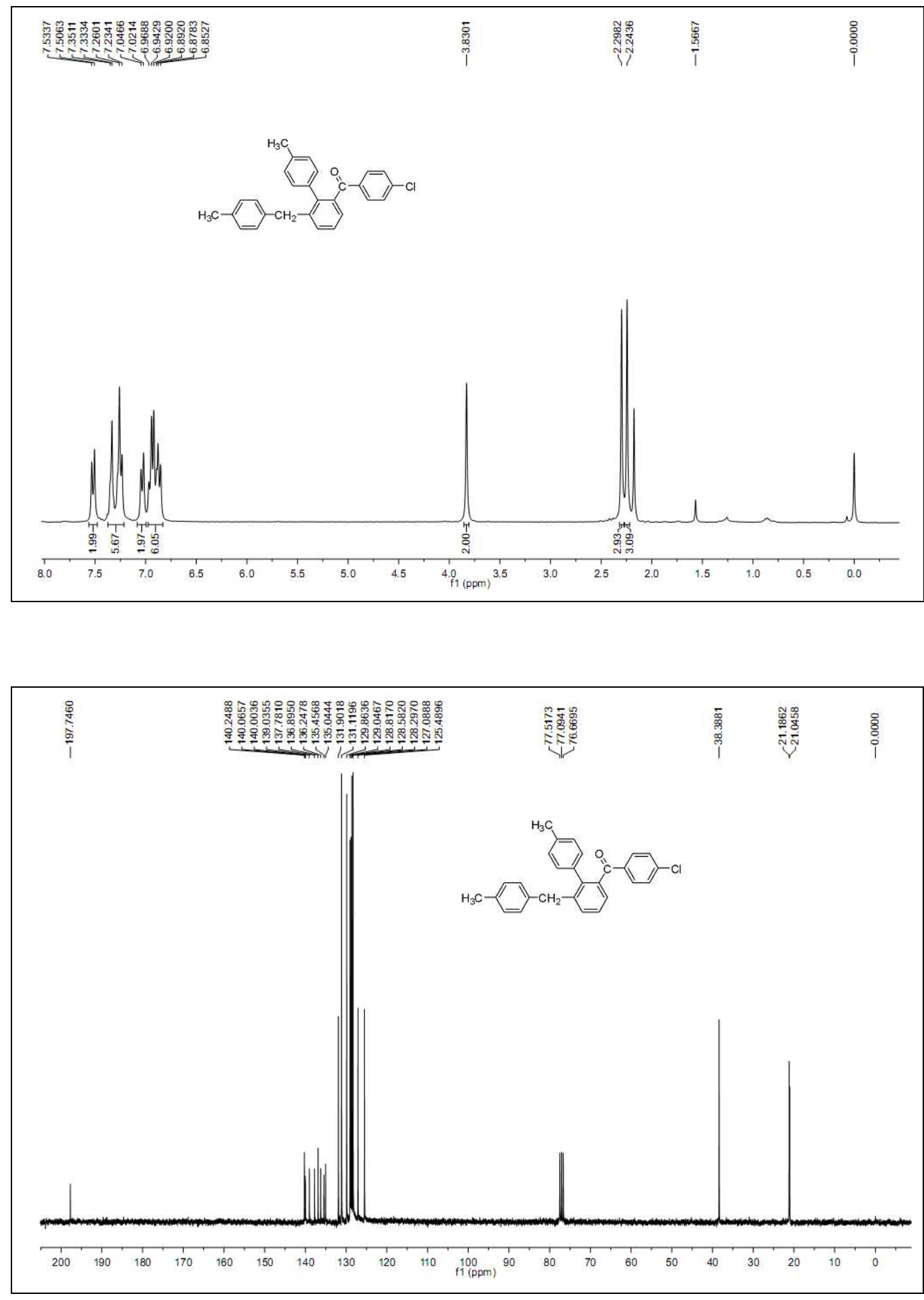
2-(4-fluorobenzyl)-4'-methyl-6-(4-chlorobenzoyl)biphenyl (5i)
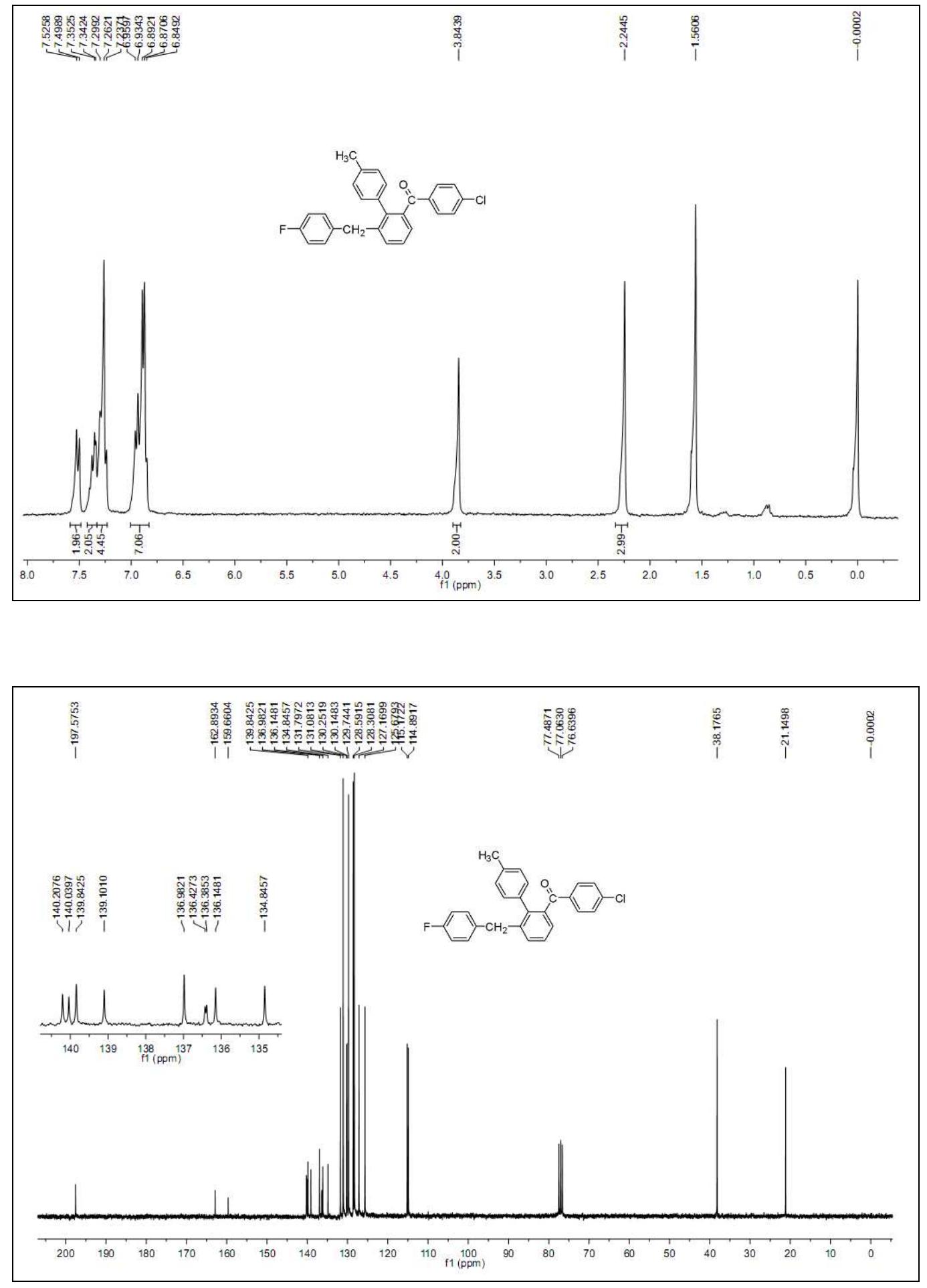